\title{
ARTICLE Lowered endogenous mu-opioid receptor availability in subclinical depression and anxiety
}

Lauri Nummenmaa $\mathbb{D}^{1,2,3}$, Tomi Karjalainen ${ }^{1}$, Janne Isojärvi $\mathbb{D}^{1}$, Tatu Kantonen $\mathbb{D}^{1,4}$, Jouni Tuisku' ${ }^{1}$, Valtteri Kaasinen $\mathbb{D}^{1,4}$, Juho Joutsa ${ }^{1,4,5}$, Pirjo Nuutila ${ }^{1,6}$, Kari Kalliokoski ${ }^{1}$, Jussi Hirvonen ${ }^{1,7}$, Jarmo Hietala $\mathbb{D}^{3,8}$ and Juha Rinne ${ }^{1}$

Major depressive disorder is associated with lowered mood, anxiety, anhedonia, sleep problems, and cognitive impairments. Many of these functions are regulated by $\mu$-opioid receptor (MOR) system. Preclinical, in vivo, and post-mortem studies have however yielded inconclusive results regarding the role of the MOR in depression and anxiety. Moreover, it is not known whether alterations in MOR are already present in subclinical depression and anxiety. In a large-scale retrospective cross-sectional study we pooled data from 135 (113 males and 22 females) healthy subjects whose brain's MOR availability was measured with positron emission tomography (PET) using an agonist radioligand $\left[{ }^{11} \mathrm{C}\right]$ carfentanil that has high affinity for MORs. Depressive and anxious symptomology was addressed with BDI-II and STAI-X questionnaires, respectively. Both anxiety and depression scores in the subclinical range were negatively associated with MOR availability in cortical and subcortical areas, notably in amygdala, hippocampus, ventral striatum, and orbitofrontal and cingulate cortices. We conclude that dysregulated MOR availability is involved in altered mood and pathophysiology of depression and anxiety disorders.

Neuropsychopharmacology (2020) 45:1953-1959; https://doi.org/10.1038/s41386-020-0725-9

\section{INTRODUCTION}

Major depressive disorder (MDD) is associated with lowered mood, diminished hedonia, sleep problems, and executive function impairments. Many of these functions are centrally regulated by opioidergic neurotransmission [1-3]. MDD has high comorbidity with anxiety disorders [4], and several studies also point towards the role of the opioid system in modulating anxiety [5-7]. Among opioid receptor (OR) classes ( $\mu, \delta$, and $\mathrm{k}$ receptors), the $\mu$-opioid receptors (MORs) mediate the effects of endogenous $\beta$-endorphins, endomorphins, enkephalins, and various exogenous opioid agonists [8]. Preclinical studies suggest that MOR agonists have both antidepressant and anxiolytic effects [3]. MOR activation with buprenorphine has antidepressant-like effects which are mediated via $\mu$-receptor partial agonism and $\mathrm{K}$ receptor antagonism [9, 10]. Clinical studies also suggest that low-dose buprenorphine treatment might be effective for depression (e.g. [11-13]), and low-dose buprenorphine therapy also leads to significant reduction in suicidality in acutely suicidal patients [14]. Uncontrolled cohort studies have further found that morphine administration following an acute trauma decreases the likelihood of subsequent post-traumatic stress disorder (PTSD), presumably via inhibition of fear conditioning following the traumatic event thus suggesting anxiolytic action of the MOR system [15-17].

In vivo imaging and post-mortem studies have yielded mixed evidence on the role of MOR in depression and anxiety. One positron emission tomography (PET) imaging study found that experimentally induced sadness decreases opioidergic neurotransmission in rostral anterior cingulate cortex (ACC) in healthy volunteers [18]. However, a more recent study reported opposite findings, with negative emotions induced by social rejection increasing opioidergic processing in amygdala and thalamus [19]. One clinical study also found increased MOR availability in the thalamus in major depression [20]; this finding was however not replicated in a subsequent study [21]. Personality trait harm avoidance (which is also a risk factor for developing affective disorders) has also been reported to correlate positively with cerebral MOR availability [22]. In PTSD, $\mu$-receptors are upregulated in the OFC and downregulated in the amygdala and ACC [23]. Data from post-mortem studies with suicide victims are mixed, with some reporting higher OR binding [24, 25], some lower binding [26], and some no differences [27] compared with controls subjects.

\section{The current study}

Altogether preclinical experiments and human pharmacological and clinical studies point towards depression and anxiety related alterations in the MOR system, whereas human in vivo imaging and post-mortem studies have yielded mixed results, possibly due to compromised statistical power. Furthermore, it is known that subthreshold depressive and anxious symptomology predict future onset of mood disorders [28], yet the contribution of the MOR system to depressive or anxious symptoms that occur prior to the first onset of a full depressive/anxious episode remain unknown. Here we tested for the association between subclinical depression and anxiety, as measured with standardized

\footnotetext{
${ }^{1}$ Turku PET Centre, University of Turku, Turku, Finland; ${ }^{2}$ Department of Psychology, University of Turku, Turku, Finland; ${ }^{3}$ Turku University Hospital University of Turku, Turku, Finland; ${ }^{4}$ Clinical Neurosciences, University of Turku and Turku University Hospital, Turku, Finland; ${ }^{5}$ Turku Brain and Mind Center, University of Turku, Turku, Finland; ${ }^{6}$ Department of Endocrinology, Turku University Hospital, Turku, Finland; ${ }^{7}$ Department of Radiology, Turku University Hospital, Turku, Finland and ${ }^{8}$ Department of Psychiatry, University of Turku and Turku University Hospital, Turku, Finland Correspondence: Lauri Nummenmaa (latanu@utu.fi)
}

Received: 9 April 2020 Revised: 8 May 2020 Accepted: 22 May 2020 Published online: 30 May 2020 
self-reports, and MOR availability in the brain in a large crosssectional register-based study. We compiled data from 135 individuals scanned with radioligand $\left[{ }^{11} \mathrm{C}\right]$ carfentanil who had also completed Beck Depression Inventory II (BDI-II; [29]) and trait anxiety scale from the state-trait anxiety inventory ((STAI-X; [30])). We show that worse subclinical symptoms of depression (i.e. BDI-II scores in the range of $0-12$ ) and anxiety are associated with lowered MOR availability, most clearly in the brain circuits governing mood and emotion regulation.

\section{MATERIALS AND METHODS}

Subjects

The sample consisted of 135, healthy individuals ( 22 females, 113 males, mean age 29, SD age 10.35 years; Tables 1 and S-1) studied with high-affinity agonist radioligand $\left[{ }^{11} \mathrm{C}\right]$ carfentanil [31], retrieved from the AIVO (http://aivo.utu.fi) database of in vivo PET images hosted at the Turku PET Centre. All subjects signed informed consent forms. Data were pooled from four scanners (GE Healthcare Discovery: 23 subjects, Siemens ECAT Siemens High Resolution Research Tool (HRRT) scans: 17 subjects, GE Healthcare Discovery 690 PET/CT scanner: 43 subjects and Philips Ingenuity PET/MR scanner: 52 subjects). All subjects had given informed consent and completed the BDI-II questionnaires as a part of the corresponding experimental protocol; subsample of 105 subjects (84 males, 21 females) had also completed the STAI-X form. Four subjects with BDI-II scores exceeding the clinical cut-off (12 points) were excluded from the sample. Because the STAI-X is not a clinical screening instrument, no cut-off could be specified; however none of the subjects had clinically diagnosed anxiety disorders. No subjects had neurologic or psychiatric diseases or currently abused alcohol or illicit drugs; subjects with medications affecting the CNS were also excluded. Seven female subjects smoked. The data were acquired between 2003 and 2018. The study protocol was approved by the Turku University Hospital Clinical Research Services Board, and the study was conducted in accordance with the declaration of Helsinki. Power analysis on prior molecular imaging studies on personality and [11C] carfentanil binding $[22,32,33]$ suggested that an expected effect size of $r=0.4$, a sample size of 71 subjects would be sufficient for establishing the predicted effects at power of 0.95 .

PET and MR image preprocessing

PET images were preprocessed using the automated PET data processing pipeline Magia [34, 35] (https://github.com/tkkarjal/ magia) running on MATLAB (The MathWorks, Inc., Natick, MA, United States). Radiotracer binding was quantified using nondisplaceable binding potential $\left(\mathrm{BP}_{\mathrm{ND}}\right)$, which is the ratio of specific binding to non-displaceable binding in the tissue [36]. This outcome measure is not confounded by differences in peripheral distribution or radiotracer metabolism. $\mathrm{BP}_{\mathrm{ND}}$ is traditionally interpreted by target molecule density $\left(B_{\max }\right)$, even though [11C] carfentanil is also sensitive to endogenous neurotransmitter

\begin{tabular}{|lll|}
\hline Table 1. $\quad$ Subject characteristics. & & \\
\hline & Males $(n=113)$ & Females $(n=22)$ \\
\hline Age & $25.23(6.23)$ & $45.50(10.70)$ \\
BDI-II score & $3.01(2.78)$ & $3.27(3.13)$ \\
STAI-X score & $33.57(7.36)$ & $32.52(5.16)$ \\
Siemens High Resolution Research & 8 & 9 \\
Tool scans & & \\
GE Healthcare Discovery 690 scans & 10 & 13 \\
GE Discovery VCT PET/CT scanner & 43 & 0 \\
Philips Ingenuity PET/MR scans & 52 & 0 \\
\hline
\end{tabular}

activation [37]. Accordingly, the $\mathrm{BP}_{\mathrm{ND}}$ for the tracer should be interpreted as density of the receptors unoccupied by endogenous ligands, i.e. receptor availability. Binding potential was calculated by applying basis function method [38] for each voxel using the simplified reference tissue model [39], with occipital cortex serving as the reference region [40]. The parametric images were spatially normalized to $\mathrm{MNI}$-space via segmentation and normalization of T1-weighted anatomical images, and finally smoothed with an 8-mm FWHM Gaussian kernel.

Previous studies have established that depression [41] and subclinical depressive symptoms are linked with compromised cerebral integrity [42]. Cerebral integrity in turn is associated with neuroreceptor-specific radiotracer uptake [43-45]. To rule out the contribution of cerebral atrophy in our results, we performed a complementary voxel-based morphometry (VBM) analysis of the $\mathrm{T} 1$ images of the subjects to control for the effect of grey matter density on radiotracer uptake. VBM was done with SPM12 (https:// www.fil.ion.ucl.ac.uk/spm/software/spm12/) which enables automated spatial normalization, tissue classification, and radiofrequency bias correction to be combined with the segmentation step. Cut-off of spatial normalization was $25 \mathrm{~mm}$ and medium affine regularization 0.01 was used. Following normalization and segmentation into GM and WM, a modulation step was incorporated to take into account volume changes caused by spatial normalization and to correct for the differences in total brain size across subjects. Finally, the segmented, normalized, and modulated GM images were smoothed using 8-mm FWHM Gaussian kernel. Regional GM densities were extracted and used to predict $[11 \mathrm{C}]$ carfentanil $\mathrm{BP}_{\mathrm{ND}}$ alongside with the $\mathrm{BDI}-\mathrm{Il}$ and STAI-X scores.

\section{Data analysis}

The population-level full-volume statistical analysis was done using SPM12. The normalized and smoothed $\mathrm{BP}_{\mathrm{ND}}$ images were entered into general linear model, where $\mathrm{BP}_{\mathrm{ND}}$ was predicted with depression and anxiety scores, respectively, while sex, age, and PET scanner were entered into the models as nuisance covariates. Statistical threshold was set at $p<0.05$, FDR corrected at cluster level. In a complementary methodological approach, the data were analyzed by averaging voxelwise $\mathrm{BP}_{\mathrm{ND}}$ within regions of interest (ROIs). Atlas-based ROIs were generated in the MOR-rich regions in the brain (amygdala, hippocampus, ventral striatum, dorsal caudate, thalamus, insula, orbitofrontal cortex (OFC), ACC, middle cingulate cortex, and posterior cingulate cortex using AAL [46] and Anatomy [47] toolboxes). The ROI data were analyzed with R statistical software (https://cran.r-project.org). Mean regional $[11 C]$ carfentanil $B P_{N D}$ and $G M$ densities from VBM were extracted for each region.

Regional effects were estimated using Bayesian hierarchical modelling using the R package BRMS [48] that uses the efficient Markov chain Monte Carlo sampling tools of RStan (https://mcstan.org/users/interfaces/rstan). We first fitted the models separately for BDI-II scores (135 subjects) and STAI-X (105 subjects) scores. Finally, we also fitted a model with both BDI-II and STAI-X scores (105 subjects) to test the unique contribution of depression and anxiety on MOR availability. We used weakly informative priors: For intercepts, we used the default of BRMS, i.e. Student's $t$ distribution with scale 3 and 10 degrees of freedom. For predictors, a Gaussian distribution with standard deviation of 1 was used to provide weak regularization. The BRMS default prior half Student's $t$-distribution with 3 degrees of freedom was used for standard deviations of group-level effects; BRMS automatically selects the scale parameter to improve convergence and sampling efficiency. The BRMS default prior LKJ [1] was used for correlations of group-level random effects. The ROI-level models were estimated using ten chains, each of which had 1000 warmup samples and 2000 post-warmup samples, thus totalling 20,000 post-warmup samples. The sampling parameters were slightly 


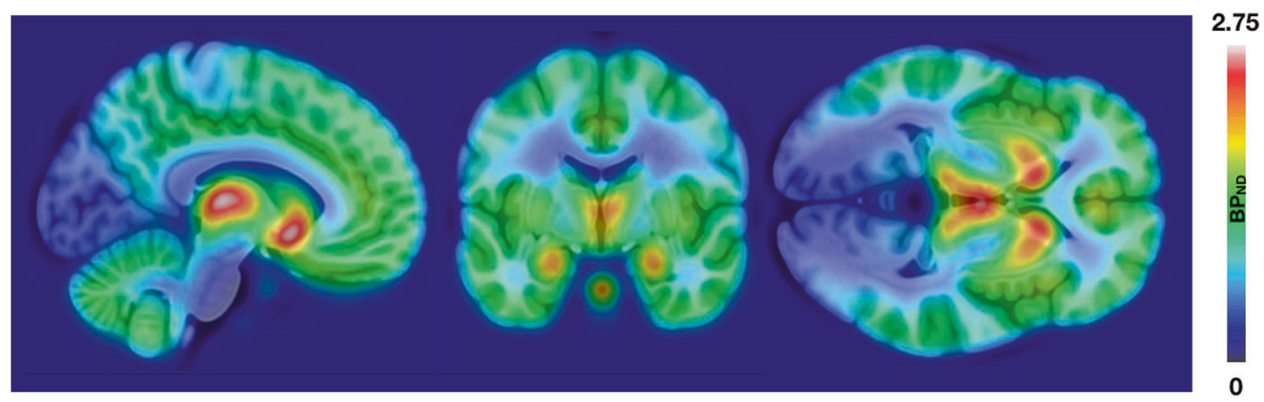

Fig. 1 Opioid receptor distribution. Averaged map of the $\left[{ }^{11} \mathrm{C}\right]$-carfentanil $\mathrm{BP}_{\mathrm{ND}}$ images in the sample $(n=135)$.

A) Negative association with BDI-II scores
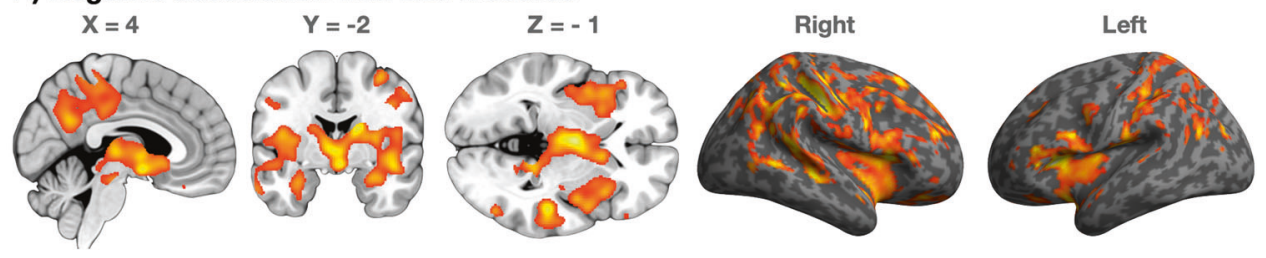

B) Negative association with STAI-X scores
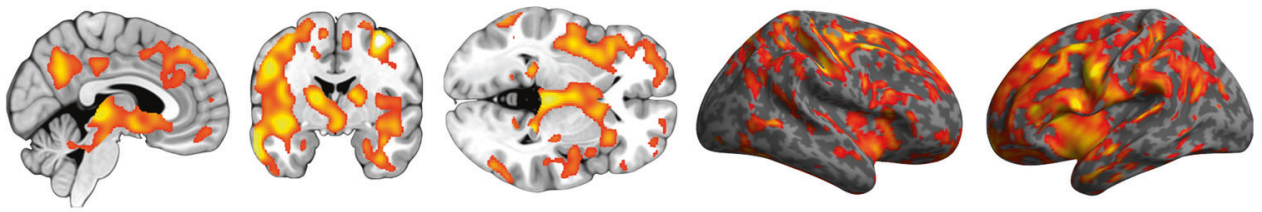

FDR

Fig. 2 Association between depressive and anxious symptoms and MOR. Regions where BDI-II (top row; $n=135$ ) and STAI-X scores (bottom row, $n=105$ ) were associated with MOR availability. The data are thresholded at $p<0.05$, FDR corrected.

modified to facilitate convergence (adapt_delta $=0.999$; max_treedepth $=20$ ). The sampling produced no divergent iterations and the Rhats were all 1.0, suggesting that the chains converged successfully. Before model estimation, continuous predictors were standardized to have zero mean and unit variance, thus making the regression coefficients comparable across the predictors. Binding potentials were log-transformed because posterior predictive checking $[49,50]$ indicated that log-transformation significantly improves model fit. The log-transformation essentially switches the model from additive to multiplicative; it also helps in model fitting because the assumption of linear additivity works poorly when the dependent variable is restricted to positive values [51].

\section{RESULTS}

Figure 1 shows the spatial distribution of MOR availability in the sample. BDI-II and STAI-X scores were positively correlated $(r=$ $0.65, p<0.001$, two-sided) but independent of age and sex ( $p: s>$ $0.05)$. Males were older than the females $(p<0.05)$. Both depression and anxiety were associated with reductions in MOR availability when controlling for age and sex (Fig. 2). For depression, these effects were widespread and bilateral. Most profound subcortical effects were found in thalamus, amygdala, hippocampus, and ventral striatum. Cortically the strongest effects were found in the insula, precuneus, and in temporoparietal regions. For anxiety, the pattern was anatomically comparable, yet the magnitude of the effect was stronger with additional clusters observed in the midcingulate and lateral prefrontal cortices. The effects for anxiety remained similar when controlling for the PET scanner. For depression, the effects remained significant in the right hemisphere. Including the four subjects with BDI-II scores $>12$ did not alter the results.

Because STAI-X scores were not available for all the subjects, we also re-ran the BDI-II analyses for the sample for which both scores were available. These data essentially replicated those for the whole sample. No regions showed positive association between MOR availability and the BDI-II and STAI scores. Due to imbalanced sex distribution, we also reanalysed the data separately in the male and female samples. The effects for the male sample were similar to those as in the whole dataset (Fig. S-1), whereas for females the associations were not significant, most likely due to compromised statistical power ( $n=22$ for BDI-II and 21 for STAI-X).

The results of the full-volume analyses were corroborated in the ROI analysis (Figs. 3, 4 and Table S-2). In general, associations between $\mathrm{BP}_{\mathrm{ND}}$ and $\mathrm{BDI}-\mathrm{Il}$ and STAI scores were negative in all the tested regions even when accounting for subjects' age, sex, regional GMD density, and PET camera. We also estimated a model that included both questionnaire scores simultaneously. In that model, the posterior distributions for the regression coefficients overlapped clearly with zero, suggesting that the contributions of the questionnaire scores are likely not independent. We also estimated a model where grey matter density was modelled with age, sex, and the questionnaire scores, and found no contributions for the BDI-II and STAI-X scales.

\section{DISCUSSION}

Our main finding was that subclinical symptoms of anxiety and depression were associated with lower MOR availability in a predominantly male sample. These effects were observed in widespread cortical and subcortical areas, most notably in 


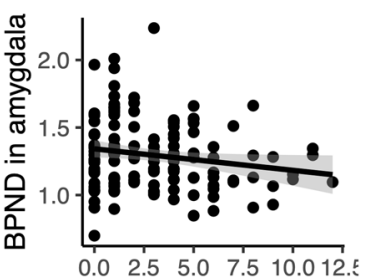

$\mathrm{BDI}$

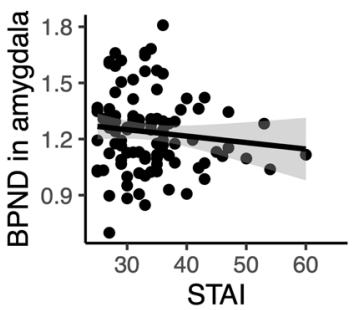

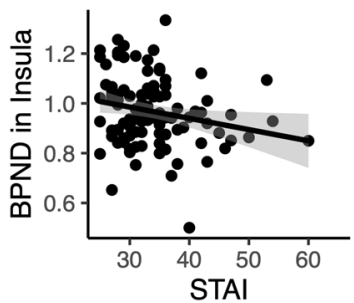

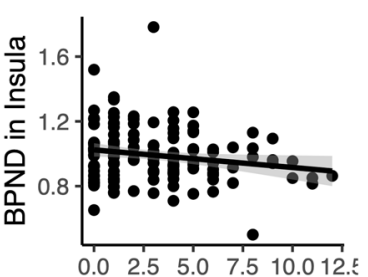

$\mathrm{BDI}$

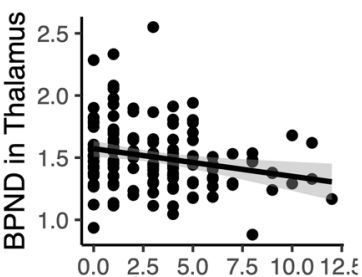

$\mathrm{BDI}$
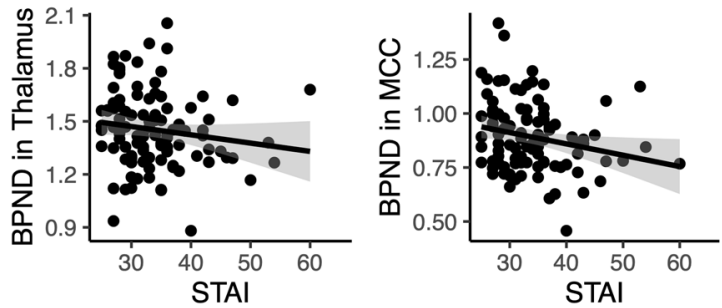

Fig. 3 Association between BDI-II and STAI-X scores on selected regions of interest. The plots show least-squares regression lines with $95 \%$ confidence intervals. Statistically significant correlations are flagged with an asterisk.

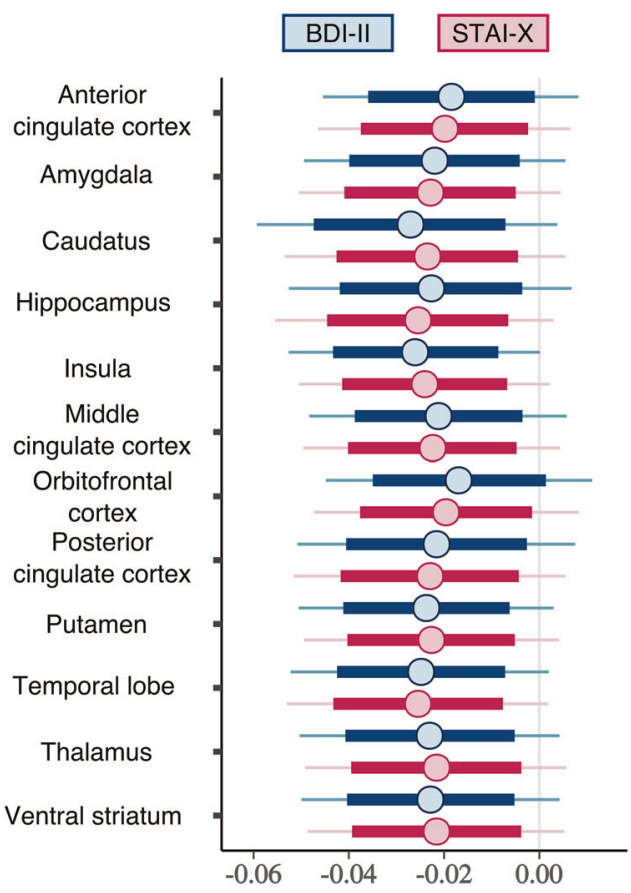

Fig. 4 Posterior distributions of the regression coefficients for the BDI-II and STAI-X scales. Thick lines show $80 \%$ posterior intervals, and the lines extend until $99 \%$ posterior intervals.

thalamus, amygdala, hippocampus, ventral striatum, and insular cortices. These effects were also independent of sex and age, and could not be explained with mesoscopic changes in cortical integrity, as quantified with VBM. As expected, depression and anxiety scores were also highly correlated $(r=0.65)$, and we could not find unique associations with the observed alterations in MOR availability in the present sample. This effect is consistent with the shared pathophysiology and concomitant comorbidity of depression and anxiety in general [4]. We did not find associations between grey matter density and the questionnaire scores, suggesting that the observed association between the MOR system and depressive/anxious symptomology cannot be explained by general structural changes in the brain. Instead, the association is more specific to the MOR system. MORs are expressed abundantly in the brain circuits governing mood and emotion regulation [1] and exogenous opioid agonists are potent modulators of mood [3]. These findings thus suggest that individual differences MOR-mediated neurotransmission might underlie sustained changes in negative affectivity, as indexed with the depressive and anxious symptomology.

Depressive and anxious symptoms are associated with low MOR availability

MOR availability, as measured with $\left[{ }^{11} \mathrm{C}\right]$ carfentanil, has high spatial autocorrelation [52] and it is thus not surprising that the depressive and anxious symptoms were associated with MOR availability in large clusters. Clear peaks were however found in thalamus, insulae, and across the cingulate cortex. Structural alterations in the dACC and insula are commonly observed across a wide range of mental illnesses, and their structural integrity is associated with cognitive function [41]. Meta-analysis of metabolic PET studies have also revealed lowered glucose metabolism in the ACC in MDD [53]. These results based on T1-weighted MR imaging or [18F]-FDG-PET are however unspecific with respect to the underlying neurobiology, whereas the present data point towards altered opioidergic neurotransmission as a potential molecular mechanism. Because ACC and insulae are involved in both affective and executive functions impaired in MDD [54, 55], this also fits with the data showing that opioidergic system modulates both executive [2] and affective functions [1]. Age is strong predictor of MOR availability $[35,56]$ and this effect-negative associations in midbrain/amygdala and positive associations in frontal and temporal cortex-was also observed in this sample (data not shown). However, age is unlikely to explain the associations between MOR, depression, and anxiety, because we adjusted for age in the statistical models, and because the correlations between age and BDI-II and STAI-X scores were not significant.

Because MOR agonists (such as $\beta$-endorphin) suppress the activity of the HPA axis by inhibiting neurons releasing corticotropin-releasing hormone in the paraventricular nucleus of the hypothalamus $[57,58]$, it is possible that dysfunctional MOR system might make an individual vulnerable to psychological stressors subsequently leading to development of depressive and anxiety disorders. As such, the present findings are also important when considering that subclinical depressive symptoms increase the risk for both depression and anxiety, whereas subclinical anxiety symptoms constitute primarily a risk factor for anxious 
psychopathology [28]: it is possible that dysregulated MOR system could be the key shared neurobiological mechanism predisposing individuals to both anxious and depressive psychopathology.

Given the centrality of opioid system in hedonia and reward [59] the present results might reflect lowered mood and/or anhedonia in the subjects scoring high on the depression and anxiety scales [60]. Indeed, human PET studies have found that MOR system responds to a variety of rewards ranging from feeding to social interaction and physical exercise [61-65]. In animal models, opioid agonists, and partial agonist buprenorphine reduce symptoms of anxiety and depression $[9,10]$ as well as alleviate the effects of psychological stressors such as separation distress [66]. Similarly in humans, fusion imaging studies with $\left[{ }^{11} \mathrm{C}\right]$ carfentanil-PET and functional magnetic resonance imaging have found that high OR availability in the striatum and limbic system suppresses haemodynamic brain responses while viewing distressing videos [67, 68]. Moreover, opioid agonists make humans evaluate external stimuli more positively [69]. Altogether these data fit with the view that MOR neurotransmission acts as an important buffer against psychological stressors. This accords with the data showing that mental health disorders are consistently associated with increased prescription opioid use, and majority of patients with opioid prescription for pain in the US also have comorbid depression and anxiety [70]. It is thus possible the lowered mood and MOR system tone might-either deliberately or inadvertently-lead to self-medication of emotional pain and dysphoria with MOR agonists, potentially aggravating the misuse potential of opioids [71].

Because none of the subjects had depression scores in the clinical range, these results do not necessarily parallel those obtained in clinically depressed patients, and future imaging studies need to address the relationship between MDD, anxiety disorders and MOR system functioning. However, our data clearly point towards the role of MOR neurotransmission in lowered mood, here indexed by depressive and anxious symptomology. These large-scale data from a subclinical sample accord with the currently limited imaging data on the role of opioidergic neurotransmission in depression in humans: First, experimentally induced negative moods deactivate the $\mu$-system in healthy volunteers, suggesting opioidergic regulation of mood [18]. Some prior small-scale clinical PET studies have also found MOR dysregulation in MDD [20] and PSTD [23]. These present data show that integrating subclinical data for well-powered statistical analysis can complement the picture drawn from clinical populations.

\section{Limitations}

Our study was based on historical data that were acquired with different PET cameras that may, despite cross-calibration, produce different binding potential estimates (See Table S-3). All images were however reanalysed using the same analysis pipeline, and potential scanner-related biases were statistically controlled for in the analyses. Not all the subjects $(n=135)$ who had completed the BDI-II had completed also STAI-X questionnaire $(n=105)$ thus the sample sizes were not equal for these two analyses. However, we also validated the effects of BDI on the sample who had completed both questionnaires, and found essentially same results as in the whole sample. Even though well-powered for the primary outcome measure, the data however do not allow establishing reliable sex differences in the depression and anxiety linked MOR expression due to imbalanced sex distribution in the sample, and due to low number of females in the sample the present results can mostly be interpreted to represent MOR function in males. Finally, the data are cross-sectional thus we cannot conclude whether the links between MOR availability and depression/anxiety scores reflect (i) genetically determined individual differences in MOR availability [72] contributing to vulnerability endophenotypes for depressive/anxious mood or (ii) downregulation of MOR neurotransmission resulting from prodromal depression or anxiety.

\section{CONCLUSIONS}

We conclude that central opioidergic neurotransmission is involved in the maintenance of depressed and anxious mood, potentially predisposing individuals to affective psychopathology. This neurobiological correlate of risk factors for MDD/anxiety disorders is relevant for pre-emptive paradigms in affective disorders. MOR system might be the critical brain system accountable for dysregulated mood in depression, and possibly further predisposing individuals for exogenous opioid abuse.

\section{FUNDING AND DISCLOSURES}

This study was supported by the Academy of Finland (grant \#294897 to LN) and Sigrid Juselius foundation. The authors declare no conflict of interest.

\section{AUTHOR CONTRIBUTIONS}

Acquired the data: TK, VK, JJ, PN, KK, JH, and JR. Analyzed the data: LN, ToK, Jl, TK, and JT. Designed the study: LN, ToK, and JR. Wrote the paper: all authors.

\section{ADDITIONAL INFORMATION}

Supplementary Information accompanies this paper at (https://doi.org/10.1038/ s41386-020-0725-9).

Publisher's note Springer Nature remains neutral with regard to jurisdictional claims in published maps and institutional affiliations.

\section{REFERENCES}

1. Nummenmaa L, Tuominen L. Opioid system and human emotions. Br J Pharmacol. 2018;175:2737-49.

2. van Steenbergen $\mathrm{H}$, Eikemo M, Leknes $\mathrm{S}$. The role of the opioid system in decision making and cognitive control: a review. Cogn Affect Behav Neurosci. 2019;19:435-58.

3. Lutz PE, Kieffer BL. Opioid receptors: distinct roles in mood disorders. Trends Neurosci. 2013;36:195-206.

4. Moffitt TE, Harrington $H$, Caspi A, Kim-Cohen J, Goldberg D, Gregory AM, et al. Depression and generalized anxiety disorder: cumulative and sequential comorbidity in a birth cohort followed prospectively to age 32 years. Arch Gen Psychiatry. 2007;64:651-60.

5. Davis M. Morphine and naloxone: effects on conditioned fear as measured with the potentiated startle paradigm. Eur J Pharmacol. 1979;54:341-7.

6. Good AJ, Westbrook RF. Effects of a microinjection of morphine into the amygdala on the acquisition and expression of conditioned fear and hypoalgesia in rats. Behav Neurosci. 1995;109:631-41.

7. Zarrindast M-R, Babapoor-Farrokhran S, Babapoor-Farrokhran S, Rezayof A. Involvement of opioidergic system of the ventral hippocampus, the nucleus accumbens or the central amygdala in anxiety-related behavior. Life Sci. 2008;82:1175-81.

8. Henriksen G, Willoch F. Imaging of opioid receptors in the central nervous system. Brain. 2008;131:1171-96.

9. Falcon E, Browne CA, Leon RM, Fleites VC, Sweeney R, Kirby LG, et al. Antidepressant-like effects of buprenorphine are mediated by kappa opioid receptors. Neuropsychopharmacology. 2016;41:2344-51.

10. Robinson SA, Erickson RL, Browne CA, Lucki I. A role for the mu opioid receptor in the antidepressant effects of buprenorphine. Behav Brain Res. 2016;319:96-103.

11. Bodkin JA, Zornberg GL, Lukas SE, Cole JO. Buprenorphine treatment of refractory depression. J Clin Psychopharmacol. 1995;15:49-57.

12. Nyhuis PW, Gastpar M, Scherbaum N. Opiate treatment in depression refractory to antidepressants and electroconvulsive therapy. J Clin Psychopharmacol. 2008;28:593-5.

13. Emrich HM, Vogt $P$, Herz A. Possible antidepressive effects of opioids: action of buprenorphine. Ann N Y Acad Sci. 1982;398:108-12.

14. Yovell Y, Bar G, Mashiah M, Baruch Y, Briskman I, Asherov J, et al. Ultra-low-dose buprenorphine as a time-limited treatment for severe suicidal ideation: a randomized controlled. Trial Am J Psychiat. 2015;173:491-8. 
15. Holbrook TL, Galarneau MR, Dye JL, Quinn K, Dougherty AL. Morphine use after combat injury in iraq and post-traumatic stress disorder. N Engl J Med. 2010;362:110-7.

16. Bryant RA, Creamer M, O'Donnell M, Silove D, McFarlane AC. A study of the protective function of acute morphine administration on subsequent posttraumatic stress disorder. Biol Psychiatry. 2009;65:438-40.

17. Saxe G, Stoddard F, Courtney D, Cunningham K, Chawla N, Sheridan R, et al. Relationship between acute morphine and the course of PTSD in children with burns. J Am Acad Child Adolesc Psychiatry. 2001;40:915-21.

18. Zubieta JK, Ketter TA, Bueller JA, Xu YJ, Kilbourn MR, Young EA, et al. Regulation of human affective responses by anterior cingulate and limbic mu-opioid neurotransmission. Arch Gen Psychiatry. 2003;60:1145-53.

19. Hsu DT, Sanford BJ, Meyers KK, Love TM, Hazlett KE, Wang H, et al. Response of the mu-opioid system to social rejection and acceptance. Mol Psychiatry. 2013;18:1211-7.

20. Kennedy SE, Koeppe RA, Young EA, Zubieta JK. Dysregulation of endogenous opioid emotion regulation circuitry in major depression in women. Arch Gen Psychiatry. 2006;63:1199-208.

21. Hsu DT, Sanford BJ, Meyers KK, Love TM, Hazlett KE, Walker SJ, et al. It still hurts: altered endogenous opioid activity in the brain during social rejection and acceptance in major depressive disorder. Mol Psychiatry. 2015;20:193-200.

22. Tuominen L, Salo J, Hirvonen J, Nagren K, Laine P, Melartin T, et al. Temperament trait Harm Avoidance associates with mu-opioid receptor availability in frontal cortex: A PET study using C-11 carfentanil. Neuroimage. 2012;61:670-6.

23. Liberzon I, Taylor SF, Phan KL, Britton JC, Fig LM, Bueller JA, et al. Altered central micro-opioid receptor binding after psychological trauma. Biol Psychiatry. 2007;61:1030-8.

24. Gross-Isseroff R, Dillon KA, Israeli M, Biegon A. Regionally selective increases in mu opioid receptor density in the brains of suicide victims. Brain Res. 1990;530:312-6.

25. Gabilondo AM, Meana JJ, García-Sevilla JA. Increased density of mu-opioid receptors in the postmortem brain of suicide victims. Brain Res. 1995;682:245-50.

26. Scarr E, Money TT, Pavey G, Neo J, Dean B. Mu opioid receptor availability in people with psychiatric disorders who died by suicide: a case control study. BMC Psychiatry. 2012;12:126.

27. Zalsman G, Molcho A, Huang Y, Dwork A, Li S, Mann JJ. Postmortem mu-opioid receptor binding in suicide victims and controls. J Neural Transm. 2005;112:949-54.

28. Karsten J, Hartman CA, Smit JH, Zitman FG, Beekman ATF, Cuijpers P, et al. Psychiatric history and subthreshold symptoms as predictors of the occurrence of depressive or anxiety disorder within 2 years. Br J Psychiatry. 2011;198:206-12.

29. Beck AT, Steer RA, Garbin MG. Psychometric properties of the Beck depression inventory: twenty-five years of evaluation. Clin Psychol Rev. 1988;8:77-100.

30. Spielberger CD, Gorsuch RL, Lushene RE. Manual for the state-trait anxiety inventory. Palo Alto, CA: Consulting Psychologists Press; 1970.

31. Frost JJ, Wagner HN Jr., Dannals RF, Ravert HT, Links JM, Wilson AA, et al. Imaging opiate receptors in the human brain by positron tomography. J Comput Assist Tomogr. 1985;9:231-6.

32. Karjalainen $T$, Tuominen $L$, Manninen $S$, Kalliokoski $K$, Nuutila $P$, Jääskeläinen IP, et al. Behavioural activation system sensitivity is associated with cerebral $\mu$-opioid receptor availability. Soc Cogn Affect Neurosci. 2016;11:1310-6.

33. Nummenmaa L, Manninen S, Tuominen L, Hirvonen J, Kalliokoski KK, Nuutila P, et al. Adult attachment style Is associated with cerebral $\mu$-opioid receptor availability in humans. Hum Brain Mapp. 2015;36:3621-8.

34. Karjalainen T, Santavirta S, Kantonen T, Tuisku J, Tuominen L, Hirvonen J, et al. Magia: robust automated modeling and image processing toolbox for PET neuroinformatics. Front Neuroinform. 2020:604835.

35. Kantonen T, Karjalainen T, Isojärvi J, Nuutila P, Tuisku J, Rinne J, et al. Interindividual variability and lateralization of $\mu$-opioid receptors in the human brain. Neuroimage. 2020;217:116922.

36. Innis RB, Cunningham VJ, Delforge J, Fujita M, Giedde A, Gunn RN, et al. Consensus nomenclature for in vivo imaging of reversibly binding radioligands. J Cereb Blood Flow Metab. 2007;27:1533-9.

37. Zubieta JK, Smith YR, Bueller JA, Xu Y, Kilbourn MR, Jewett DM, et al. Regional mu opioid receptor regulation of sensory and affective dimensions of pain. Science. 2001:293:311-5

38. Gunn RN, Lammertsma AA, Hume SP, Cunningham VJ. Parametric imaging of ligand-receptor binding in PET using a simplified reference region model. Neurolmage. 1997;6:279-87.

39. Lammertsma AA, Hume SP. Simplified reference tissue model for PET receptor studies. Neurolmage. 1996;4:153-8.

40. Frost JJ, Douglass KH, Mayberg HS, Dannals RF, Links JM, Wilson AA. Multicompartmental analysis of $[11 \mathrm{C}]$-carfentanil binding to opiate receptors in humans measured by positron emission tomography. J Cereb Blood Flow Metab. 1989;9:398-409.
41. Goodkind M, Eickhoff SB, Oathes DJ, Jiang Y, Chang A, Jones-Hagata LB, et al. Identification of a common neurobiological substrate for mental illness. JAMA Psychiatry. 2015;72:305-15.

42. Hayakawa YK, Sasaki H, Takao H, Mori H, Hayashi N, Kunimatsu A, et al. Structural brain abnormalities in women with subclinical depression, as revealed by voxelbased morphometry and diffusion tensor imaging. J Affect Disord. 2013;144:263-8.

43. Kraus $C$, Hahn A, Savli M, Kranz GS, Baldinger $P$, Hoeflich $A$, et al. Serotonin-1A receptor binding is positively associated with gray matter volume-a multimodal neuroimaging study combining PET and structural MRI. Neuroimage. 2012;63:1091-8.

44. Woodward ND, Zald DH, Ding Z, Riccardi P, Ansari MS, Baldwin RM, et al. Cerebral morphology and dopamine D2/D3 receptor distribution in humans: a combined [18F]fallypride and voxel-based morphometry study. Neuroimage. 2009:46:31-8.

45. Manninen S, Karjalainen T, Tuominen L, Hietala J, Kaasinen V, Joutsa J, et al. Cerebral grey matter density is associated with neuroreceptor and neurotransporter availability: a combined PET and MRI study. 2020. https://www. biorxiv.org/content/10.1101/2020.01.29.924530v1.full.

46. Tzourio-Mazoyer N, Landeau B, Papathanassiou D, Crivello F, Etard O, Delcroix N, et al. Automated anatomical labeling of activations in SPM using a macroscopic anatomical parcellation of the MNI MRI single-subject brain. Neuroimage. 2002;15:273-89.

47. Eickhoff SB, Stephan KE, Mohlberg H, Grefkes C, Fink GR, Amunts K, et al. A new SPM toolbox for combining probabilistic cytoarchitectonic maps and functional imaging data. Neuroimage. 2005;25:1325-35.

48. Bürkner P-C. brms: an R package for Bayesian multilevel models using Stan. J Stat Softw. 2017;80:1-28.

49. Gelman A, Carlin JB, Stern HS, Dunson DB, Vehtari A, Rubin DB. Bayesian data analysis. Chapman and Hall/CRC; Boca Raton, FL, 2013.

50. Gabry J, Simpson D, Vehtari A, Betancourt M, Gelman A. Visualization in Bayesian workflow. J R Stat Soc Ser A. 2019;182:389-402.

51. Gelman A, Hill J. Data analysis using regression and multilevel/hierarchical models. Cambridge University Press; New York, NY, 2006.

52. Tuominen L, Nummenmaa L, Keltikangas-Järvinen L, Raitakari O, Hietala J. Mapping neurotransmitter networks with PET: an example on serotonin and opioid systems. Hum Brain Mapp. 2014;35:1875-84.

53. Su L, Cai Y, Xu Y, Dutt A, Shi S, Bramon E. Cerebral metabolism in major depressive disorder: a voxel-based meta-analysis of positron emission tomography studies. BMC Psychiatry. 2014;14:321.

54. Craig AD. How do you feel? Interoception: the sense of the physiological condition of the body. Nat Rev Neurosci. 2002;3:655.

55. Hamilton JP, Etkin A, Furman DJ, Lemus MG, Johnson RF, Gotlib IH. Functional neuroimaging of major depressive disorder: a meta-analysis and new integration of baseline activation and neural response. Data Am J Psychiatry. 2012;169:693-703.

56. Zubieta JK, Dannals RF, Frost JJ. Gender and age influences on human brain muopioid receptor binding measured by PET. Am J Psychiatry. 1999;156:842-8.

57. Grisel JE, Bartels JL, Allen SA, Turgeon VL. Influence of beta-Endorphin on anxious behavior in mice: interaction with EtOH. Psychopharmacology. 2008:200:105-15.

58. Wand GS, Mangold D, El Deiry S, McCaul ME, Hoover D. Family history of alcoholism and hypothalamic opioidergic activity. Arch Gen Psychiatry. 1998;55:1114-9.

59. Hnasko TS, Sotak BN, Palmiter RD. Morphine reward in dopamine-deficient mice. Nature. 2005;438:854-7.

60. Fox ME, Lobo MK. The molecular and cellular mechanisms of depression: a focus on reward circuitry. Mol Psychiatry 2019;24:1798-815.

61. Manninen S, Tuominen L, Dunbar RIM, Karjalainen T, Hirvonen J, Arponen E, et al. Social laughter triggers endogenous opioid release in humans. J Neurosci. 2017;37:6125-31.

62. Tuulari JJ, Tuominen L, de Boer FE, Hirvonen J, Helin S, Nuutila P, et al. Feeding releases endogenous opioids in humans. J Neurosci. 2017;37:8284-91.

63. Burghardt PR, Rothberg AE, Dykhuis KE, Burant CF, Zubieta JK. Endogenous opioid mechanisms are implicated in obesity and weight loss in humans. J Clin Endocrinol Metab. 2015;100:3193-201.

64. Saanijoki T, Tuominen L, Tuulari JJ, Nummenmaa L, Arponen E, Kalliokoski K, et al. Opioid release after high-intensity interval training in healthy human subjects. Neuropsychopharmacology. 2017;43:246-54.

65. Nummenmaa L, Tuominen L, Dunbar R, Hirvonen J, Manninen S, Arponen E, et al. Social touch modulates endogenous $\mu$-opioid system activity in humans. Neuroimage. 2016;138:242-7.

66. Herman BH, Panksepp J. Effects of morphine and naloxone on separation distress and approach attachment: Evidence for opiate mediation of social affect. Pharmacol Biochem Behav. 1978;9:213-20. 
67. Karjalainen T, Seppala K, Glerean E, Karlsson HK, Lahnakoski JM, Nuutila P, et al. Opioidergic regulation of emotional arousal: a combined PET-fMRI study. Cereb Cortex. 2019;29:4006-16.

68. Karjalainen T, Karlsson HK, Lahnakoski JM, Glerean E, Nuutila P, Jaaskelainen IP, et al. Dissociable roles of cerebral mu-opioid and type 2 dopamine receptors in vicarious pain: a combined PET-fMRI study. Cereb Cortex. 2017:27:1-10.

69. Heiskanen $T$, Leppä $M$, Suvilehto J, Akural E, Larinkoski T, Jääskeläinen IP, et al. The opioid agonist remifentanil increases subjective pleasure during emotional stimulation. Br J Anaesthesiol. 2019;122:E216-E219.
70. Davis MA, Lin LA, Liu H, Sites BD. Prescription opioid use among adults with mental health disorders in the United States. J Am Board Fam Med. 2017;30:407.

71. Peciña M, Karp JF, Mathew S, Todtenkopf MS, Ehrich EW, Zubieta J-K. Endogenous opioid system dysregulation in depression: implications for new therapeutic approaches. Mol Psychiatry 2019;24:576-87.

72. Weerts EM, McCaul ME, Kuwabara H, Yang XJ, Xu XQ, Dannals RF, et al. Influence of OPRM1 Asn(40)Asp variant (A118G) on C-11 carfentanil binding potential: preliminary findings in human subjects. Int J Neuropsychopharmacol. 2013;16: 47-53. 